\title{
Autonomic cardiac regulation and morpho-physiological responses to eight week training preparation in junior soccer players
}

\author{
Michal Botek, Jakub Krejčí*, and Radim Weisser \\ Faculty of Physical Culture, Palacký University, Olomouc, Czech Republic
}

Copyright: (C) 2014 M. Botek et al. This is an open access article licensed under the Creative Commons Attribution License (http://creativecommons.org/licenses/by/4.0/).

Background: Training preparation in soccer is thought to improve body composition and performance level, especially the maximal aerobic capacity $\left(\mathrm{VO}_{2}\right.$ max). However, an enhancement in performance may be attenuated by the increase of fatigue. Heart rate variability (HRV) as a non-invasive index of autonomic nervous system (ANS) activity has been considered to be a sensitive tool in fatigue assessment. Objective: This study was focused to evaluate the response of ANS activity and morpho-physiological parameters to eight week training preparation. Methods: Study included 12 trained soccer players aged $17.2 \pm 1.2$ years. Athletes underwent pre- and post-preparation testing that included the ANS activity assessment by spectral analysis of HRV in supine and upright position. Further, body composition was analyzed via electrical bio-impedance method and physiological parameters were assessed during maximal stress tests. ANS activity and subjective feeling of fatigue was assessed continuously within subsequent weeks of preparation. Results: No significant differences in all HRV variables within weeks were found. Pre vs. post analyses revealed a significant $(p<.05)$ increase in body weight, fat free mass, body mass index, and peak power. A significant decline in mean maximal heart rate (HR) and resting HR at standing was identified at the end of preparation. Since no significant changes between pre- post-preparation in the mean $\mathrm{VO}_{2}$ max occurred, the positive correlation between the individual change in $\mathrm{VO}_{2}$ max and the vagally related HRV [supine LnHF $(r=.78)$, Ln $\operatorname{rMSSD}(r=.63)$, and the standing LnHF $(r=.73, p<.05)]$ was found. Conclusions: This study showed that an 8 week training program modified particularly fat free mass and short-term endurance, whereas both the autonomic cardiac regulation and the feeling of fatigue remained almost unaffected. Standing position seems to be more sensitive in terms of the HR response in relation to fatigue perception than supine.

Keywords: heart rate variability, adaptation, exercise, vagal activity, body composition, fatigue

\section{Introduction}

Success during a soccer match is dependent, amongst other factors, on technical ability, tactics and a high level of physical conditioning, especially repeated sprint ability and aerobic endurance (Stølen, Chamari, Castagna, \& Wisløff, 2005). Previously, it was demonstrated that there is an association between distance covered during a match and maximal oxygen uptake ( $\left.\mathrm{VO}_{2} \max \right)$ (Bangsbo, 1994). Elite junior players have been shown to cover $10 \mathrm{~km}$ on average during a match (Helgerud, Engen, Wisloff, \& Hoff, 2001) with studies reporting that $\mathrm{VO}_{2}$ max values of junior players range from 55 to $65 \mathrm{ml} \cdot \mathrm{kg}^{-1} \cdot \mathrm{min}^{-1}$ (Da Silva, Bloomfield,

\footnotetext{
* Address for correspondence: Jakub Krejčí, Department of Natural Sciences in Kinanthropology, Faculty of Physical Culture, Palacký University, tř. Míru 115, 77111 Olomouc, Czech Republic.E-mail: jakub.krejci@upol.cz
}

\& Marins, 2008; Impellizzeri, Rampinini, \& Marcora, 2005; Reilly, Bangsbo, \& Franks, 2000). Besides $\mathrm{VO}_{2}$ max, running economy also takes an important part in aerobic endurance determination. For example, Helgerud et al. (2001) showed the 6.7\% increase in running economy after 8 week of aerobic training in junior soccer players.

Standardized aerobic training programs have been shown to increase $\mathrm{VO}_{2}$ max by $25 \%$ in untrained subjects (Lortie et al., 1984). However, to date, the literature has repeatedly reported heterogeneous responses in $\mathrm{VO}_{2} \max$ to the same aerobic training program that ranged from decreases to over $40 \%$ enhancements in $\mathrm{VO}_{2} \max$ (Boutcher, Park, Dunn, \& Boutcher, 2013; Hautala, Kiviniemi, \& Tulppo, 2009; Hautala et al., 2003; Kohrt et al., 1991; Vesterinen et al., 2013). Some authors have suggested that the inter-individual variation in adaptive responses to training is related to the individual training status ("trainability"). They 
have argued that "trainability" is associated with the resting level of autonomic nervous system (ANS) activity, particularly vagal activity (Botek, McKune, Krejčí, Stejskal, \& Gába, 2014; Boutcher et al., 2013; Hautala, Kiviniemi, \& Tulppo, 2009; Hautala et al., 2003; Kiviniemi, Hautala, Kinnunen, \& Tulppo, 2007; Vesterinen et al., 2013). The use of non-invasive tools, such as resting heart rate (HR) and heart rate variability (HRV), for the assessment of training status has become very popular among coaches, and athletes, respectively (Buchheit, 2014).

Spectral analysis (SA) of RR intervals to determine HRV is commonly accepted as a non-invasive method for ANS activity assessment (Akselrod et al., 1981). Both, high frequency (HF) oscillations in RR intervals (Akselrod et al., 1981; Pichot et al., 2000), and a root of mean square successive differences (rMSSD) (Buchheit, 2014) have been suggested to reflect the cardiac vagal outflow, while reciprocal changes between vagal and sympathetic activity are evaluated via analysis of low frequency power LF (Task Force of the European Society of Cardiology and the North American Society of Pacing and Electrophysiology, 1996) and LF/HF (Ori, Monir, Weiss, Sayhouni, \& Singer, 1992), respectively. A supine position is widely used for ANS activity assessment in athletes (Aubert, Seps, \& Beckers, 2003; Buchheit, 2014; Hedelin, Bjerle, \& Henriksson-Larsén, 2001; Pichot et al., 2000) despite the finding that a plateau in HF power in individuals with a low resting HR may occur (Goldberger, Challapalli, Tung, Parker, \& Kadish, 2001; Kiviniemi et al., 2007; Plews, Laursen, Stanley, Kilding, \& Buchheit, 2013). Therefore, to measure changes in the vagal activity in trained athletes, it has been recommended that they are measured in an upright position (Kiviniemi et al., 2007).

So far, there are two studies in young soccer players where autonomic response was assessed only in supine position in order to identify the change in training status (Buchheit, Chivot et al., 2010), and the level of fatigue (Bricout, Dechenaud, \& Favre-Juvin, 2010), respectively. With respect to limitations in HRV analysis mentioned above, autonomic response to training doses in soccer players was assessed in both supine, and in the upright position.

The main purpose of this study was to evaluate the response of both the $\mathrm{HR}$, and autonomic activity to cumulative training stress in supine, and in standing position, respectively. A secondary aim of this study was to assess the changes in anthropometrical and physiological variables before and after training preparation. In addition, we also investigated the relation between the level of autonomic activity and changes in $\mathrm{VO}_{2}$ max, and subjective feelings of fatigue. It was hypothesized that the upright position may be more sensitive in the response of both the HR, and the autonomic cardiac regulation to cumulative stress than the supine position in trained young soccer players during training preparation.

\section{Methods}

\section{Participants}

Sixteen soccer players who played in the first Czech junior soccer league were volunteered to participate in this study. In order to be included to the research players supposed to: 1) perform pre- and post-testing; 2) participate in more than $90 \%$ of training sessions; and 3 ) be free of any medical contraindications to perform maximal exercise tests. Based on including criteria, four players had to be excluded during the research. Basic characteristics of the twelve players are presented in Table 1 together with results. The research design was approved by the Ethics Committee of the Faculty of Physical Culture, Palacký University. An informed consent was signed by each of the players or parents of a player who were at that time under eighteen years old prior participation in the research.

\section{Experimental procedures}

Two weeks prior to the start of the winter preparation athletes underwent preliminary medical screening to determine the presence of any limitations that may prevent them from performing maximal stress testing. The subjects were required to avoid eating, drinking coffee, tea and/or any substance affecting the ANS activity for a minimum of two hours before the preliminary measurements. In addition, they were requested to avoid vigorous physical activity and alcohol for 48 hours before testing. After medical screening all players were allowed to undergo a pre-laboratory testing protocol, including resting HRV measurement, basic anthropological assessment, and maximal stress test, respectively. Before measurement, all players were out of any training sessions for at least 6 weeks due to the transitive period in soccer periodization. Postlaboratory testing was performed 5 days after the final friendly match. Each player had two days rest before post-testing.

During training preparation, players completed 56 total training units (TU), including regeneration units, and 8 friendly matches. All players had a playing time of 90 minutes per match. A standard trainings were then replaced with the high intensity interval drills (HIID), including $2 \times 15 \mathrm{~m}$ sprint, 9 repetitions, 3 sets, passive rest of 30 seconds between repetitions, and 5 minutes active recovery between sets, respectively, that were performed with maximal voluntary 
Table 1

Results of bioelectrical impedance analysis, maximal incremental exercise test and HRV analysis before (Pre) and after (Post) eight-week training program

\begin{tabular}{|c|c|c|c|c|c|c|}
\hline \multirow[b]{2}{*}{ Variable (unit) } & \multicolumn{2}{|c|}{ Mean \pm SD } & \multicolumn{4}{|c|}{ Difference Post versus Pre } \\
\hline & Pre & Post & Mean & $95 \% \mathrm{CI}$ & $p$ & $d$ \\
\hline \multicolumn{7}{|l|}{ Anthropological variables } \\
\hline Body mass (kg) & $68.2 \pm 8.6$ & $69.7 \pm 7.8$ & 1.5 & {$[0.1,2.8]$} & .041 & $0.67^{+}$ \\
\hline $\operatorname{BMI}\left(\mathrm{kg} \cdot \mathrm{m}^{-2}\right)$ & $20.9 \pm 2.0$ & $21.4 \pm 1.7$ & 0.5 & {$[0.0,0.8]$} & .042 & $0.66^{+}$ \\
\hline Fat percentage (\%) & $12.1 \pm 4.5$ & $11.4 \pm 4.1$ & -0.7 & {$[-2.3,1.0]$} & .398 & -0.25 \\
\hline Fat mass $(\mathrm{kg})$ & $8.4 \pm 3.9$ & $8.1 \pm 3.4$ & -0.3 & {$[-1.6,0.9]$} & .572 & -0.17 \\
\hline FFM (kg) & $59.9 \pm 6.8$ & $61.7 \pm 6.3$ & 1.8 & {$[0.4,3.1]$} & .014 & $0.84^{+}$ \\
\hline \multicolumn{7}{|l|}{ Physiological variables } \\
\hline HRmax (beats $\cdot \min ^{-1}$ ) & $200 \pm 10$ & $195 \pm 8$ & -5 & {$[-7,-2]$} & .002 & $-1.14^{+}$ \\
\hline $\mathrm{VO}_{2} \max \left(\mathrm{mL} \cdot \mathrm{min}^{-1} \cdot \mathrm{kg}^{-1}\right)$ & $53.0 \pm 3.9$ & $53.1 \pm 5.9$ & 0.1 & {$[-1.8,2.1]$} & .845 & 0.06 \\
\hline $\operatorname{Pmax}\left(\mathrm{W} \cdot \mathrm{kg}^{-1}\right)$ & $5.92 \pm 0.38$ & $6.12 \pm 0.38$ & 0.20 & {$[0.01,0.39]$} & .038 & $0.68^{+}$ \\
\hline \multicolumn{7}{|l|}{ HRV variables } \\
\hline $\mathrm{HR}_{\text {Standing }}$ (beats $\cdot \mathrm{min}^{-1}$ ) & $103 \pm 16$ & $95 \pm 16$ & -8 & {$[-14,0]$} & .040 & $-0.67^{+}$ \\
\hline $\operatorname{LnLF}_{\text {Standing }}\left(\mathrm{ms}^{2}\right)$ & $5.6 \pm 1.1$ & $6.2 \pm 1.3$ & 0.6 & {$[-0.1,1.2]$} & .107 & $0.51^{+}$ \\
\hline $\mathrm{LnHF}_{\text {Standing }}\left(\mathrm{ms}^{2}\right)$ & $4.4 \pm 1.6$ & $4.6 \pm 1.6$ & 0.2 & {$[-0.3,0.8]$} & .368 & 0.27 \\
\hline $\mathrm{Ln} \mathrm{LF} / \mathrm{HF}_{\text {Standing }}$ & $1.2 \pm 1.0$ & $1.5 \pm 0.8$ & 0.3 & {$[-0.4,1.0]$} & .334 & 0.29 \\
\hline Ln rMSSD ${ }_{\text {Standing }}(\mathrm{ms})$ & $2.54 \pm 0.68$ & $2.76 \pm 0.82$ & 0.22 & {$[-0.03,0.46]$} & .081 & $0.56^{+}$ \\
\hline $\mathrm{HR}_{\text {Supine }}\left(\right.$ beats $\cdot \mathrm{min}^{-1}$ ) & $65 \pm 12$ & $63 \pm 11$ & -2 & {$[-5,2]$} & .377 & -0.27 \\
\hline $\operatorname{LnLF}_{\text {Supine }}\left(\mathrm{ms}^{2}\right)$ & $6.1 \pm 1.3$ & $6.5 \pm 1.3$ & 0.4 & {$[-0.4,1.2]$} & .265 & 0.34 \\
\hline $\operatorname{LnHF}_{\text {Supine }}\left(\mathrm{ms}^{2}\right)$ & $7.4 \pm 1.6$ & $7.2 \pm 1.4$ & -0.2 & {$[-0.6,0.3]$} & .401 & -0.25 \\
\hline $\mathrm{Ln} \mathrm{LF} / \mathrm{HF}_{\text {Supine }}$ & $-1.3 \pm 1.1$ & $-0.7 \pm 0.9$ & 0.6 & {$[-0.1,1.3]$} & .089 & $0.54^{+}$ \\
\hline $\operatorname{Ln~rMSSD}_{\text {Supine }}(\mathrm{ms})$ & $4.28 \pm 0.85$ & $4.32 \pm 0.63$ & 0.04 & {$[-0.26,0.35]$} & .756 & 0.09 \\
\hline
\end{tabular}

Note. $95 \% \mathrm{CI}=95 \%$ confidence interval, $p=$ significance of paired t-test, $d=$ Cohen's $d$ effect size, BMI = body mass index, $\mathrm{FFM}=$ fat free mass, $\mathrm{HRmax}=$ maximum heart rate, $\mathrm{VO}_{2} \max =$ maximum oxygen uptake per $\mathrm{kg}, \mathrm{Pmax}=$ maximum mechanical power, Standing $=$ standing phase of orthoclinostatic maneuver, Supine $=$ supine phase of orthoclinostatic maneuver, HR $=$ heart rate, $\mathrm{LnLF}=$ natural logarithm of low-frequency power, $\mathrm{LnHF}=$ natural logarithm of high-frequency power, $\mathrm{Ln} \mathrm{LF} / \mathrm{HF}=\mathrm{natural}$ logarithm of low-frequency/high-frequency ratio, Ln rMSSD = natural logarithm of root mean square successive difference of RR intervals. ${ }^{+}$moderate or large effect size.

effort, and covered distance ( $\sim 800 \mathrm{~m})$ was appropriately equal to a mean distance that players usually encountered during a match in a sprint (Stølen et al., 2005). The time frame of whole experiment, number of TU per week with a brief description of TU content, and the placement of both the morning HRV measurements, and HIID during preparation is presented in Figure 1, and in Table 2.

The HRV assessment during preparation was executed between 7:30 am to 10:00 am at the beginning of each training week (Monday). The ANS activity assessments were performed in a quiet room (ambient temperature of $22-24{ }^{\circ} \mathrm{C}$ ). Before each measurement, the players sat for a 10 minutes resting period. A timemodified orthoclinostatics challenge of ANS activity (Botek, Krejčí, Neuls, \& Novotný, 2013) test was then performed. The athletes were instructed to maintain their same dietary and hydration habits throughout the study. Before each morning's HRV assessment began, players were asked to fill in a questioner that consisted of 6 points non-validated scale of subjective feeling of fatigue ( 0 - none, 1 - mild; 2 - moderate, 3 - somewhat high, 4 - high, 5 - very high fatigue).

\section{Anthropometrical assessment}

Each soccer player had their body height (BSM370, Biospace, Seoul, South Korea) and body mass (BM) measured during pre- and post-preparation period as well as fat free mass (FFM), fat mass (FM), and percentage of body fat (\% BF) by using the Tanita BC-418 MA bioelectrical impedance analysis device (Tanita, Tokyo, Japan).

\section{Maximal stress test}

The player performed a graded maximal stress test on a treadmill (Lode Valiant, Groningen, Netherlands) in 


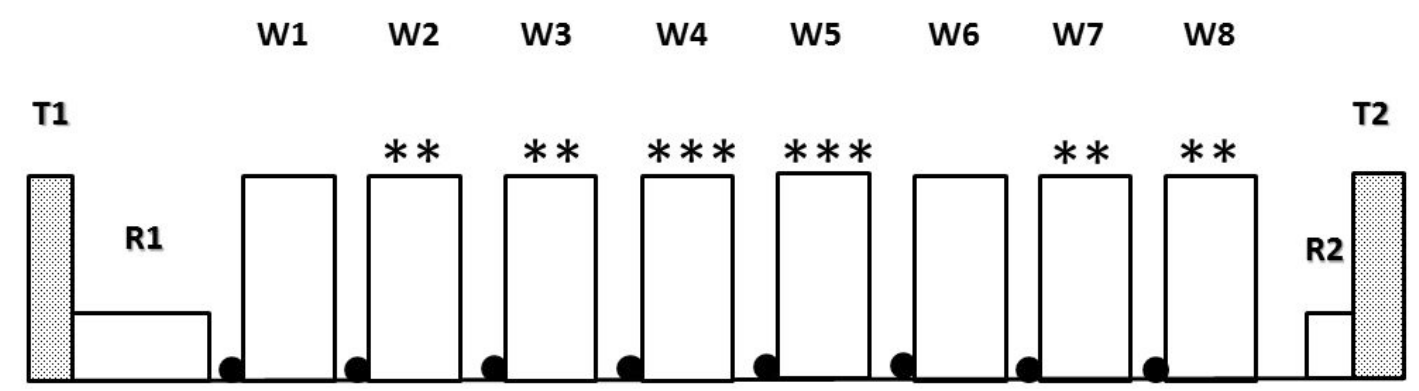

Figure 1. Illustration of experimental design. $\mathrm{T} 1=$ pre-preparatory testing, $\mathrm{T} 2=$ post-preparatory testing, $\mathrm{R} 1$ = two weeks resting period, $\mathrm{R} 2$ = two days resting period, $\mathrm{W}(1-8)=$ training week, * = number of high intensity training drills, $\bullet=$ heart rate variability assessment.

Table 2

Content and distribution of physical exercises within preparation

\begin{tabular}{|c|c|c|c|c|c|c|c|}
\hline \multirow[t]{2}{*}{ Content } & \multicolumn{2}{|c|}{ Physical exercises } & \multicolumn{2}{|c|}{ Non-condition training } & \multicolumn{3}{|c|}{ Condition training } \\
\hline & F-match & Training & $\mathrm{T}-\mathrm{T}$ & REC & Speed & Strength & S-E \\
\hline $1^{\text {st }}$ & 1 & 6 & 1 & 1 & 0 & 1 & 3 \\
\hline $2^{\text {nd }}$ & 1 & 6 & 2 & 1 & 1 & 1 & 1 \\
\hline $3^{\text {rd }}$ & 1 & 7 & 3 & 1 & 1 & 0 & 2 \\
\hline $4^{\text {th }}$ & 1 & 6 & 2 & 1 & 0 & 1 & 2 \\
\hline $5^{\text {th }}$ & 1 & 7 & 3 & 1 & 1 & 1 & 1 \\
\hline $6^{\text {th }}$ & 1 & 6 & 3 & 1 & 0 & 0 & 2 \\
\hline $7^{\text {th }}$ & 1 & 7 & 3 & 1 & 1 & 0 & 2 \\
\hline $8^{\text {th }}$ & 1 & 6 & 3 & 1 & 0 & 1 & 1 \\
\hline Sum PE & 8 & 51 & 20 & 8 & 4 & 5 & 14 \\
\hline Percentage & 100 & 100 & 39.2 & 15.7 & 7.8 & 9.8 & 27.5 \\
\hline
\end{tabular}

Note. $\quad$ F-match $=$ friendly match, $\mathrm{T}-\mathrm{T}=$ technical-tactical training, $\mathrm{REC}=$ recovery training, $\mathrm{S}-\mathrm{E}=$ speed-endurance training, $\mathrm{PE}=$ physical exercise.

order to obtain $\mathrm{VO}_{2}$ max; HRmax, and maximal power output (Pmax), respectively. The protocol consisted of 5 minutes warm-up period $\left(4\right.$ minutes at $\left.8 \mathrm{~km} \cdot \mathrm{h}^{-1}\right)$ followed by an increased in $5 \%$ elevation for 1 minute in given speed. Thereafter, the speed increased for $1 \mathrm{~min}-$ ute to $12 \mathrm{~km} \cdot \mathrm{h}^{-1}$ and every following minute increased in $1 \mathrm{~km} \cdot \mathrm{h}^{-1}$ until subject reached maximal speed equal to $16 \mathrm{~km} \cdot \mathrm{h}^{-1}$. Then, an inclination increased by $2.5 \%$ every min until exhaustion. Breath-by-breath ventilation and gas exchange (Geratherm system, Bad Kissingen, Germany) were continuously analyzed during the exercise with the data averaged to 30 seconds for analysis. The $\mathrm{VO}_{2} \max$ was recorded as the highest oxygen consumption value in the final 30 seconds of the test. HR responses (Polar, Kempele, Finland) were monitored continuously during maximal stress test. Pmax was established indirectly according to following formula (American College of Sports Medicine, 1986):
Load $[\mathrm{W}]=$ weight $\cdot[($ speed $\cdot 0.2)+($ grade $\cdot$ speed .

$$
\cdot 0.9)+3.5] / 10.5
$$

Note. speed $=$ speed in meters $/$ minutes, grade $=$ grade numerical, grade percentage must be calculated $(5 \%$ is equivalent to 0.05 ), weight $=$ weight in kilograms .

\section{Heart rate variability analysis}

To determine the resting HR and HRV variables, the ECG signal was measured at a sampling frequency of $1000 \mathrm{~Hz}$ using a VarCor PF7 diagnostic device (DIMEA Group, Olomouc, Czech Republic). Each recording lasted approximately 15 minutes while the four players simultaneously performed the timemodified orthoclinostatics maneuver (supine-standing-supine) on a tilt table. It is important to note that the first 60 second lasting supine position served as the standard condition setting before the orthostatic challenge (Botek et al., 2013). 
The ECG record was examined, and all premature ventricular contractions, missing beats, and any artefacts were manually filtered. A set of 300 artefact-free subsequent RR intervals was obtained from each phase. A SA HRV was used to assess the ANS activity and was performed using the Fast Fourier Transform. The SA incorporated a sliding 256 points Hanning window and a Coarse-Graining Spectral Analysis algorithm (Yamamoto \& Hughson, 1991). The power spectra were quantified by integrating the area under the power spectral density curve. Two frequency bands were used: LF from 0.05 to $0.15 \mathrm{~Hz}$ and $\mathrm{HF}$ from 0.15 to $0.50 \mathrm{~Hz}$. A time domain variable, the root mean square successive difference of RR intervals (rMSSD) was also used.

\section{Statistical methods}

All statistical analyses were performed by using STATISTICA (Version 12; StatSoft, Tulsa, OK, USA) and MATLAB (Version 8.2; MathWorks, Natick, MA, USA). Normal Gaussian distribution of the analyzed data was verified by the Kolmogorov-Smirnov test. Because the data was not-normally distributed, a natural logarithm transformation ( $\mathrm{Ln}$ ) was applied to obtain a normal distribution of following variables:

- $\operatorname{LnLF}=\operatorname{Ln}(\mathrm{LF})$,

- $\operatorname{LnHF}=\mathrm{Ln}(\mathrm{HF})$,

- $\mathrm{Ln} \mathrm{LF} / \mathrm{HF}=\mathrm{Ln}(\mathrm{LF} / \mathrm{HF})$,

- $\operatorname{Ln}$ rMSSD = Ln(rMSSD $)$.

Paired $t$-test was conducted to evaluate the differences in all variables obtained during the pre-training and the post-training laboratory examination. Variables were further evaluated by calculating the Cohen's $d$ effect size. One-way analysis of variance (ANOVA) for repeated measures was used to evaluate the changes on each HRV variable during eight week training program. The Pearson's coefficient of correlation $(r)$ was calculated to assess the relationships between change of $\mathrm{VO}_{2}$ max and $\mathrm{HRV}$ variables. In all analyses, $p<.05$ was considered to be statistically significant. Data are expressed as means $\pm \mathrm{SD}$.

\section{Results}

\section{Physical characteristics of participants}

Table 1 shows that a significant increase in BM $(p=.041)$, BMI $(p=.042)$, and FFM $(p=.014)$ was observed after the preparation compared to pre-training values. Moreover, effect size for BM $(d=0.67)$, BMI $(d=0.66)$, and FFM $(d=0.84)$ suggested a moderate to high practical significance. With regards of physiological variables, there was a significant reduction $(p=.002)$ in HRmax and significant increase in
Pmax $(p=.038)$ after the preparation compared to pre-testing values. In this regards, effect size value for HRmax $(d=-1.14)$, and Pmax $(d=0.68)$ suggested a moderate to high practical significance.

\section{Heart rate, heart rate variability, feeling of fatigue}

From Table 1 is further evident that the mean HR in standing significantly decreased $(p=.040)$ in post-testing compared to pre-testing period, and the practical significance for this change $(d=-0.67)$ was established as moderate. In standing, the non-significant increase in $\operatorname{LnLF}(p=.107)$, and $\operatorname{rMSSD}(p=.081)$ in posttesting compared to pre-testing period was found, however, effect size for $\operatorname{LnLF}(d=0.51), \operatorname{rMSSD}(d=0.56)$ indicated a moderate practical significance. Non-significant decrease $(p=.089)$ in supine $\mathrm{Ln} \mathrm{LF} / \mathrm{HF}$ was detected in post-testing period, however, effect size for Ln LF/HF $(d=0.54)$ suggested a moderate practical significance.

Table 3 shows that there are no significant differences between subsequent weeks of training preparation either in the HR, or HRV variables in both testing position. Also, the level of subjective feeling of fatigue did not vary significantly between subsequent weeks of training preparation.

Table 3

ANOVA of HRV variables and morning fatigue feeling during eight subsequent weeks

\begin{tabular}{|c|c|c|}
\hline \multirow[b]{2}{*}{ Variable (unit) } & \multicolumn{2}{|c|}{ ANOVA } \\
\hline & $p$ & Partial $\eta^{2}$ \\
\hline $\mathrm{HR}_{\text {Standing }}$ (beats $\cdot \mathrm{min}^{-1}$ ) & .760 & .05 \\
\hline $\mathrm{LnLF}_{\text {Standing }}\left(\mathrm{ms}^{2}\right)$ & .645 & .06 \\
\hline $\operatorname{LnHF}_{\text {Standing }}\left(\mathrm{ms}^{2}\right)$ & .122 & .13 \\
\hline $\mathrm{Ln} \mathrm{LF} / \mathrm{HF}_{\text {Standing }}$ & .120 & .13 \\
\hline Ln rMSSD Standing $_{\text {(ms) }}$ & .215 & .11 \\
\hline $\mathrm{HR}_{\text {Supine }}$ (beats $\cdot \mathrm{min}^{-1}$ ) & .932 & .03 \\
\hline $\operatorname{LnLF}_{\text {Supine }}\left(m s^{2}\right)$ & .990 & .02 \\
\hline $\operatorname{LnHF}_{\text {Supine }}\left(\mathrm{ms}^{2}\right)$ & .897 & .04 \\
\hline $\mathrm{Ln} \mathrm{LF} / \mathrm{HF}_{\text {Supine }}$ & .942 & .03 \\
\hline $\operatorname{Ln~rMSSD}_{\text {Supine }}(\mathrm{ms})$ & .660 & .06 \\
\hline Morning fatigue feeling & .363 & .09 \\
\hline
\end{tabular}

Note. $p=$ significance of ANOVA, partial $\eta^{2}=$ partial etasquared, Standing $=$ standing phase of orthoclinostatic maneuver, Supine $=$ supine phase of orthoclinostatic maneuver, $\mathrm{HR}=$ heart rate, $\mathrm{LnLF}=$ natural logarithm of low-frequency power, LnHF = natural logarithm of high-frequency power, $\mathrm{Ln} \mathrm{LF} / \mathrm{HF}$ = natural logarithm of low-frequency/high-frequency ratio, $\mathrm{Ln}$ rMSSD = natural logarithm of root mean square successive difference of RR intervals. 


\section{Correlation analysis}

An individual relative improvement (0.4-6.9\%) and relative deterioration (1.7-14.0\%) in $\mathrm{VO}_{2} \max$ after training preparation were detected in six and six soccer players, respectively. As demonstrates Figure 2, Pearson correlation analysis revealed a relationship between the relative change in $\mathrm{VO}_{2}$ max and the mean supine LnHF ( $r=.78, p=.003)$, Ln $\operatorname{rMSSD}(r=.63$, $p=.029)$, and the mean standing LnHF $(r=.73$, $p=.007)$. From Table 4 is clear that except the relationship $(r=.59, p=.042)$ between the feeling of fatigue and the standing HR, no further significant correlation between subjective feeling of morning fatigue and HRV variables was observed.

\section{Discussion}

The main findings of this study are following: i) significant changes in BM and FFM; ii) significant increase
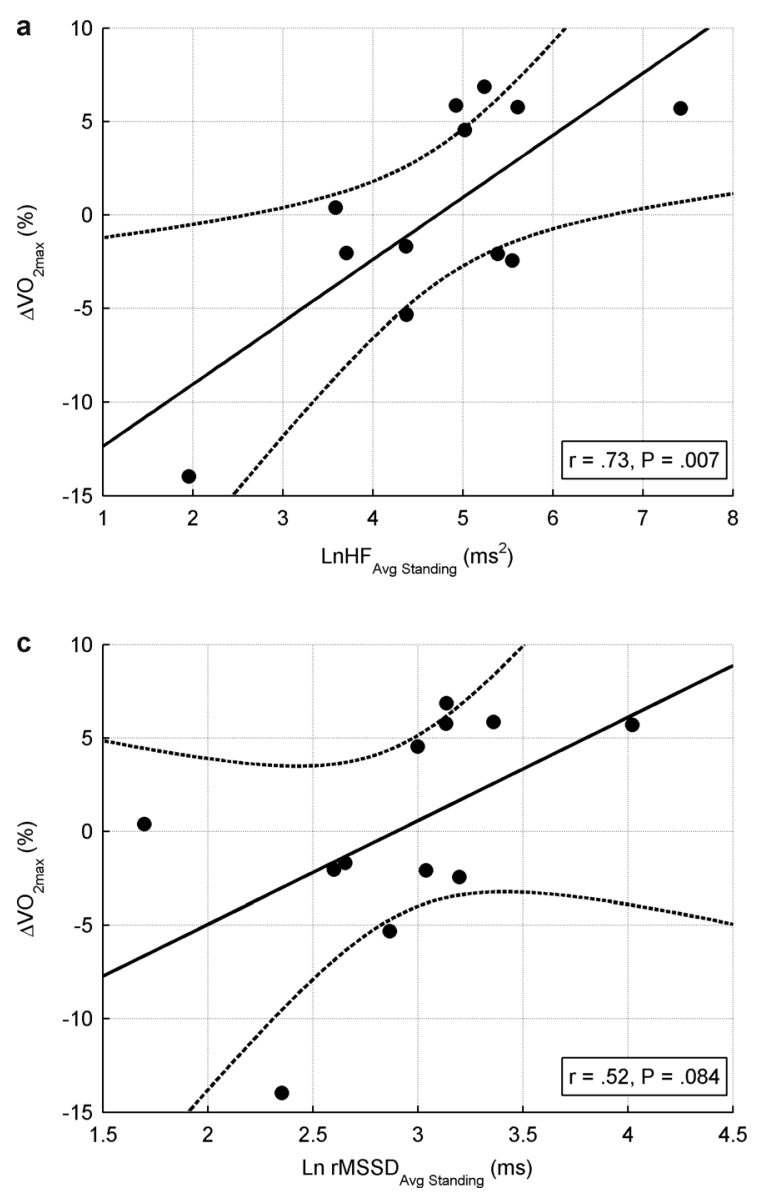

in Pmax during maximal stress test; iii) a positive relationship between vagal activity level and the relative change of $\mathrm{VO}_{2} \max$ in supine and standing position.

We found a significant increase in mean of BM, FFM, and BMI, while the fat mass remained unchanged after preparation. An increase in FFM has been associated with high intensity training and/or resistant training that induced a muscular enlargement (Goldberg, Etlinger, Goldspink, \& Jablecki, 1975). On the other hand, a minimal impact of training on the fat mass in our study was probably also due to its optimal level that is comparable level to standards for age matched group of soccer players (Gil, Gil, Ruiz, Irazusta, \& Irazusta, 2007).

Although HRmax is not traditionally considered as the indicator of training status (Brooks, Fahey, \& White, 1995; Hickson, Hagberg, Ehsani, \& Holloszy, 1981) in contrast to HRrest in the response to aerobic training (Guyton, 2000), our research revealed a markedly decrease in HRmax after training. In this regards,
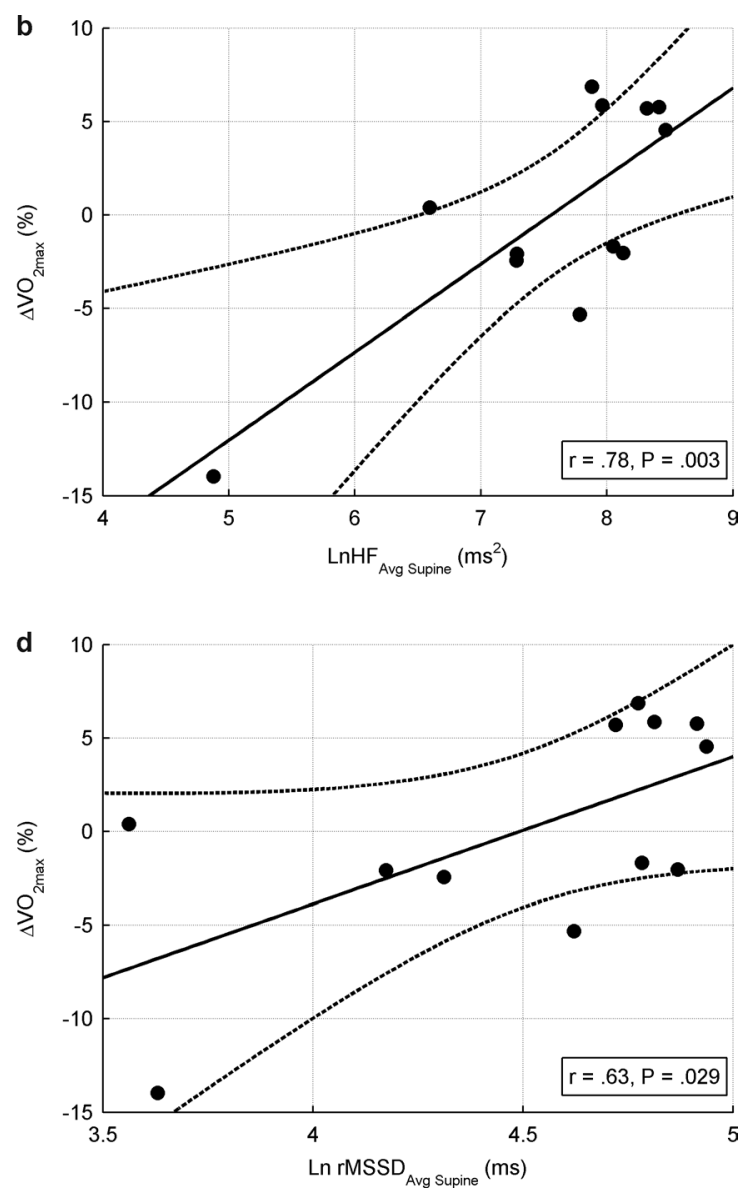

Figure 2. Correlation analysis between relative change of oxygen uptake and the mean of natural logarithm of high-frequency power ( $a$ and $b$ ), the mean of natural logarithm of root mean square successive difference of RR intervals $\left(\mathrm{c}\right.$ and d). $\Delta \mathrm{VO}_{2} \max =$ relative change of oxygen uptake after eight-week training program (percentage post versus pre), Standing = standing phase of orthoclinostatic maneuver, Supine = supine phase of orthoclinostatic maneuver. Dashed lines denote $95 \%$ confidence interval. 
Table 4

Correlation analysis between morning feeling of fatigue and the mean of HRV variables

\begin{tabular}{|c|c|c|}
\hline \multirow[b]{2}{*}{ Variable (unit) } & \multicolumn{2}{|c|}{ Correlation } \\
\hline & $r$ & $p$ \\
\hline $\mathrm{HR}_{\text {Standing }}$ (beats $\cdot \mathrm{min}^{-1}$ ) & .59 & .042 \\
\hline $\operatorname{LnLF}_{\text {Standing }}\left(\mathrm{ms}^{2}\right)$ & -.32 & .317 \\
\hline $\operatorname{LnHF}_{\text {Standing }}\left(\mathrm{ms}^{2}\right)$ & -.37 & .232 \\
\hline $\mathrm{Ln} \mathrm{LF/HF}$ Standing & .25 & .425 \\
\hline Ln rMSSD ${ }_{\text {Standing }}(\mathrm{ms})$ & -.35 & .268 \\
\hline $\mathrm{HR}_{\text {Supine }}$ (beats $\cdot \mathrm{min}^{-1}$ ) & .26 & .425 \\
\hline $\operatorname{LnLF}_{\text {Supine }}\left(\mathrm{ms}^{2}\right)$ & -.13 & .685 \\
\hline $\operatorname{LnHF}_{\text {Supine }}\left(\mathrm{ms}^{2}\right)$ & -.29 & .354 \\
\hline $\mathrm{Ln} \mathrm{LF} / \mathrm{HF}_{\text {Supine }}$ & .25 & .425 \\
\hline $\operatorname{Ln~rMSSD}_{\text {Supine }}(\mathrm{ms})$ & -.18 & .586 \\
\hline
\end{tabular}

Note. $r$ = Pearson's correlation coefficient, $p=$ significance of correlation, Standing = standing phase of orthoclinostatic maneuver, Supine $=$ supine phase of orthoclinostatic maneuver, $\mathrm{HR}=$ heart rate, $\mathrm{LnLF}=$ natural logarithm of low-frequency power, $\mathrm{LnHF}=$ natural logarithm of high-frequency power, $\mathrm{Ln} \mathrm{LF} / \mathrm{HF}$ = natural logarithm of low-frequency/high-frequency ratio, $\mathrm{Ln}$ rMSSD = natural logarithm of root mean square successive difference of RR intervals.

Zavorsky (2000) in review study showed that HRmax is reduced following regular aerobic training in endurance athletes, when decline in HRmax may be related to both the plasma volume expansion and enhanced baroreflex function, and the decrease in $\beta$-adrenergic receptor number and density.

Soccer players in our study achieved significantly higher level of Pmax during maximal exercise post-test compared to pre-testing level, while the $\mathrm{VO}_{2}$ max level remained unchanged. It seems that training preparation primarily focused on the both speed-endurance and explosive strength may lead among others to the enhancement in the work economy. Our results are in agreement with the study of Hickson, Dvorak, Gorostiaga, Kurowski, and Foster (1988) who showed that an incorporation the strength drills into endurance program resulted in an increase of $13 \%$ in short-term endurance (time to exhaustion on the treadmill) together with unchanged $\mathrm{VO}_{2}$ max level. On the other hand, Botek et al. (2010) demonstrated a significant increase in $\mathrm{VO}_{2}$ max after 5 weeks training in soccer players who performed both strength, and endurance drills.

A wide range $(-14.0 \%$ to $+6.9 \%)$ in $\mathrm{VO}_{2} \max$ response among soccer players to similar training load was identified in this study. In this regards has been assumed that factor such as age, gender, ethnicity, baseline fitness status, nutrition, sleep, prior training, and genetics may explain the individual responses in $\mathrm{VO}_{2}$ max to endurance training (Bouchard \& Rankinen, 2001; Buchheit, 2014; Vollaard et al., 2009). We found a positive correlation between $\Delta \mathrm{VO}_{2} \max$ and the mean morning level of vagally modulated HRV components in supine and upright position, respectively, measured throughout the training preparation. Based on these results, one would suggest that athletes who display a higher level of vagal activity obtained either in supine or upright position may be more sensitive for the improving in aerobic capacity than athletes with lower vagal activity. Unfortunately, the physiological mechanisms underlying the link between the vagal activity and aerobic fitness enhancement remained so far unclear. Hautala et al. (2009) assumed that, including genetic factors, there is also possible a mechanistic link between cardiac vagal activity and training response, whereas cardiovascular system of subject with higher vagal activity level may have better adaptation response to external stress stimuli. Our findings are in line with Vesterinen et al. (2013) who reported that high vagal activity at the baseline was related with good adaptation response to high intensity training, whereas low vagal activity seems to indicate poor training adaptability possibly reduced by a state of fatigue. In former study Hedelin, Bjerle, and Henriksson-Larsén (2001) considered vagal activity level as an inherited quality that determines the limits on further increase in $\mathrm{VO}_{2} \max$ of trained subjects. Based on mentioned, it could be assumed that the assessment of vagal activity level by SA HRV may be a useful indicator of the soccer player's ability for improvement in $\mathrm{VO}_{2} \max$ after the training.

Our results further indicate no significant changes in all HRV variables assessed either in supine or upright position within subsequent training weeks. In addition, the level of subjective feeling of fatigue also did not differ within weeks in players. Bricout, Dechenaud, and Favre-Juvin (2010) in young soccer players study showed a significantly decrease vagal activity in a day after competitive match compared to a day after one resting day. Thus, we feel that more than 24 hours after training week in our study seems to be a sufficient time for ANS activity restoration in well trained young soccer players. In another study has been shown that there is no relation between total active time (trainings and matches) and HRV parameters, whereas the high cardio-respiratory fitness levels decreases the day-today variation in HRV in young soccer players (Buchheit, Mendez-Villanueva, Quod, Poulos, \& Bourdon, 2010). Although fatigue was correlated with both HRV, and HR, only positive correlation between subjective feeling of fatigue and HR in standing was found. From a practical point of view, it seems to be favorable 
regarding the fatigue the HR assesses in upright position rather than in supine.

According to our results, there are no significant changes in HRV variables obtained either in the supine or in the upright position after the eight weeks. However, after preparation a significant decrease in mean HR in standing position was revealed, while no significant changes at mean HR in supine after training compared to the preliminary level occurred. Changes in resting HR without modification in autonomic cardiac activity after training have been already described. Catai et al. (2002) found changes in the resting HR that were not followed by alternation in HRV variables, and suggested that the resting bradycardia induced by the training in young subjects is much more related to intrinsic alterations in sinus node than in efferent vagal-sympathetic modulation.

In the light of results is evident that a higher density of HRV measurement during a training week in well trained young soccer players is requested to reveal the ANS responses to training doses. For example Buchheit (2014) recommended the HRV measurements at least 3-4 times per week in individually exercised endurance athletes. According to same author, for team sports may be more suitable monitor the heart rate recovery rather then HRV level, because more frequent application HRV measurement could be related with higher technical and logistical complications in the daily training practice.

\section{Conclusions}

In conclusion, this study showed that standard 8 week training program in young soccer players influenced particularly the mean FFM, and the mean Pmax, whereas autonomic cardiac regulation remained unchanged. It was further revealed that an individual $\mathrm{VO}_{2}$ max response to training load was associated with the vagal activity level in both the standing, and the supine position. In addition, standing position seems to be more sensitive in terms of the HR response in relation to fatigue perception than supine position in young soccer players.

\section{Acknowledgment}

The study has been supported by the research grant from the Ministry of Education, Youth and Sports of the Czech Republic (No. MSM 6198959221) "Physical Activity and Inactivity of the Inhabitants of the Czech Republic in the Context of Behavioral Changes". We would like to thank our already graduated students
Pavel Rychlý, Ondřej Sucháček, and Petr Mach for their assistance in collection HRV data in this research.

\section{References}

Akselrod, S., Gordon, D., Ubel, F. A., Shannon, D. C., Berger, A. C., \& Cohen, R. J. (1981). Power spectrum analysis of heart rate fluctuation: A quantitative probe of beat to beat cardiovascular control. Science, 213, 220-222.

American College of Sports Medicine. (1986). Guidelines for exercise testing and prescription (3rd ed.). Philadelphia, PA: Lea \& Febiger.

Aubert, A. E., Seps, B., \& Beckers, F. (2003) Heart rate variability in athletes. Sports Medicine, 33, 889-919.

Bangsbo, J. (1994). The physiology of soccer-with special reference to intense intermittent exercise. Acta Physiologica Scandinavica, 619, 1-155.

Botek, M., McKune, A. J., Krejčí, J., Stejskal, P., \& Gába, A. (2014). Change in performance in response to training load adjustment based on autonomic activity. International Journal of Sports Medicine, 35, 482-488.

Botek, M., Krejčí, J., Neuls, F., \& Novotný, J. (2013). Effect of modified method of autonomic nervous system activity assessment on results of heart rate variability analysis. Acta Universitatis Palackianae Olomucensis. Gymnica, 43(2), 39-46.

Botek, Z., Gába, A., Lehnert, M., Přidalová, M., Vařeková, R., Botek, M., \& Langer, F. (2010). Condition and body constitution of soccer players in category U19 before and after completing a preparatory period. Acta Universitatis Palackianae Olomucensis. Gymnica, 40(2), 47-54.

Bouchard, C., \& Rankinen, T. (2001). Individual differences in response to regular physical activity. Medicine \& Science in Sports \& Exercise, 33(Suppl. 6), S446-S451.

Boutcher, S. H., Park, Y., Dunn, S. L., \& Boutcher, Y. N. (2013). The relationship between cardiac autonomic function and maximal oxygen uptake response to high-intensity intermittent-exercise training. Journal of Sports Sciences, 31, 1024-1029.

Bricout, V. A., Dechenaud, S., \& Favre-Juvin, A. (2010). Analyses of heart rate variability in young soccer players: the effects of sport activity. Autonomic Neuroscience, 154, 112-116.

Brooks, G. A., Fahey, T. D., \& White, T. D. (1995). The heart. In G. A. Brooks, T. D. Fahey, \& T. D. White (Eds.), Exercise physiology: Human bioenergetics and its applications (pp. 243-259). Mayfield: Mountain View.

Buchheit, M. (2014). Monitoring training status with HR measures: Do all roads lead to Rome? Frontiers in Physiology, 27, 73.

Buchheit, M., Chivot, A., Parouty, J., Mercier, D., Al Haddad, H., Laursen, P. B., \& Ahmaidi, S. (2010). Monitoring endurance running performance using cardiac parasympathetic function. European Journal of Applied Physiology, 108, 1153-1167.

Buchheit, M., Mendez-Villanueva, A., Quod, M. J., Poulos, N., \& Bourdon, P. (2010). Determinants of the variability of heart rate measures during a competitive period in young soccer players. European Journal of Applied Physiology, 109, 869-878. 
Catai, A. M., Chacon-Mikahil, M. P., Martinelli, F. S., Forti, V. A., Silva, E., Golfetti, R., ... Gallo-Junior, L. (2002). Effects of aerobic exercise training on heart rate variability during wakefulness and sleep and cardiorespiratory responses of young and middle-aged healthy men. Brazilian Journal of Medical and Biological Research, 35, 741-752.

Da Silva, C. D., Bloomfield, J., \& Marins, J. C. (2008). A review of stature, body mass and maximal oxygen uptake profiles of U17, U20 and first division players in Brazilian soccer. Journal of Sports Science \& Medicine, 7, 309-319.

Gil, S. M., Gil, J., Ruiz, F., Irazusta, A., \& Irazusta, J. (2007). Physiological and anthropometric characteristics of young soccer players according to their playing position: Relevance for the selection process. Journal of Strength and Conditioning Research, 21, 438-445.

Goldberg, A. L., Etlinger, J. D., Goldspink, D. F., \& Jablecki, C. (1975). Mechanism of work-induced hypertrophy of skeletal muscle. Medicine and Science in Sports, 7, 185-198.

Goldberger, J. J., Challapalli, S., Tung, R., Parker, M. A., \& Kadish A. H. (2001). Relationship of heart rate variability to parasympathetic effect. Circulation, 103, 1977-1983.

Guyton, A. C. (2000). Textbook of medical physiology (10th ed.). Philadelphia, PA: Saunders.

Hautala, A. J., Kiviniemi, A. M., \& Tulppo, M. P. (2009). Individual responses to aerobic exercise: The role of the autonomic nervous system. Neuroscience and Biobehavioral Reviews, 33, 107-115.

Hautala, A. J., Mäkikallio, T. H., Kiviniemi, A., Laukkanen, R. T., Nissilä, S., Huikuri, H. V., \& Tulppo, M. P. (2003). Cardiovascular autonomic function correlates with the response to aerobic training in healthy sedentary subjects. American Journal of Physiology. Heart and Circulation Physiology, 285, 1747-1752.

Hedelin, R., Bjerle, P., \& Henriksson-Larsén, K. (2001). Heart rate variability in athletes: Relationship with central and peripheral performance. Medicine \& Science in Sports \& Exercise, 33, 1394-1398.

Helgerud, J., Engen, L. C., Wisloff, U., \& Hoff, J. (2001). Aerobic endurance training improves soccer performance. Medicine \& Science in Sports \& Exercise, 33, 1925-1931.

Hickson, R. C., Dvorak, B. A., Gorostiaga, E. M., Kurowski, T. T., \& Foster, C. (1988). Potential for strength and endurance training to amplify endurance performance. Journal of Applied Physiology, 65, 2285-2290.

Hickson, R. C., Hagberg, J. M., Ehsani, A. A., \& Holloszy, J. O. (1981). Time course of the adaptive responses of aerobic power and heart rate to training. Medicine \& Science in Sports \& Exercise, 13, 17-20.

Impellizzeri, F. M., Rampinini, E., \& Marcora, S. M. (2005). Physiological assessment of aerobic training in soccer. Journal of Sports Sciences, 23, 583-592.

Kiviniemi, A. M., Hautala, A. J., Kinnunen, H., \& Tulppo, M. P. (2007). Endurance training guided individually by daily heart rate variability measurements. European Journal of Applied Physiology, 101, 743-751.

Kohrt, W. M., Malley, M. T., Coggan, A. R., Spina, R. J., Ogawa, T., Ehsani, A. A., ... Holloszy, J. O. (1991). Effects of gender, age, and fitness level on response of $\mathrm{VO}_{2} \max$ to training in 60-71 years old. Journal of Applied Physiology, 71, 2004-2011.

Lortie, G., Simoneau, J. A., Hamel, P., Boulay, M. R., Landry, F., \& Bouchard, C. (1984). Responses of maximal aerobic power and capacity to aerobic training. International Journal of Sports Medicine, 5, 232-236.

Ori, Z., Monir, G., Weiss, J., Sayhouni, X., \& Singer, D. H. (1992). Heart rate variability. Frequency domain analysis. Cardiology Clinics, 10, 499-537.

Pichot, V., Roche, F., Gaspoz, J. M., Enjolras, F., Antoniadis, A., Minini, P., ... Barthélémy, J. C. (2000). Relation between heart rate variability and training load in middledistance runners. Medicine \& Science in Sports \& Exercise, 32, 1729-1736.

Plews, D. J., Laursen, P. B., Stanley, J., Kilding, A. E., \& Buchheit, M. (2013). Training adaptation and heart rate variability in elite endurance athletes: opening the door to effective monitoring. Sports Medicine, 43, 773-781.

Reilly, T., Bangsbo, J., \& Franks, A. (2000). Anthropometric and physiological predispositions for elite soccer. Journal of Sports Sciences, 18, 669-683.

Stølen, T., Chamari, K., Castagna, C., \& Wisløff, U. (2005). Physiology of soccer: An update. Sports Medicine, 35, 501-536.

Task Force of the European Society of Cardiology and the North American Society of Pacing and Electrophysiology. (1996). Heart rate variability: standards of measurement, physiological interpretation and clinical use. Circulation, 93, 1043-1065.

Vesterinen, V., Häkkinen, K., Hynynen, E., Mikkola, J., Hokka, L., \& Nummela, A. (2013). Heart rate variability in prediction of individual adaptation to endurance training in recreational endurance runners. Scandinavian Journal of Medicine \& Science in Sports, 23, 171-180.

Vollaard, N. B., Constantin-Teodosiu, D., Fredriksson, K., Rooyackers, O., Jansson, E., Greenhaff, P. L., ... Sundberg, C. J. (2009). Systematic analysis of adaptations in aerobic capacity and submaximal energy metabolism provides a unique insight into determinants of human aerobic performance. Journal of Applied Physiology, 106, 1479-1486.

Yamamoto, Y., \& Hughson, R. L. (1991). Coarse-graining spectral analysis: New method for studying heart rate variability. Journal of Applied Physiology, 71, 1143-1150.

Zavorsky, G. S. (2000). Evidence and possible mechanisms of altered maximum heart rate with endurance training and tapering. Sports Medicine, 29, 13-26. 